\title{
FRACTURAS PÉLVICAS Y DE CADERA INFANTIL
}

Porque la mejor forma de aprender las consecuencias de nuestros traumatismos es que se rompan ellos solos.

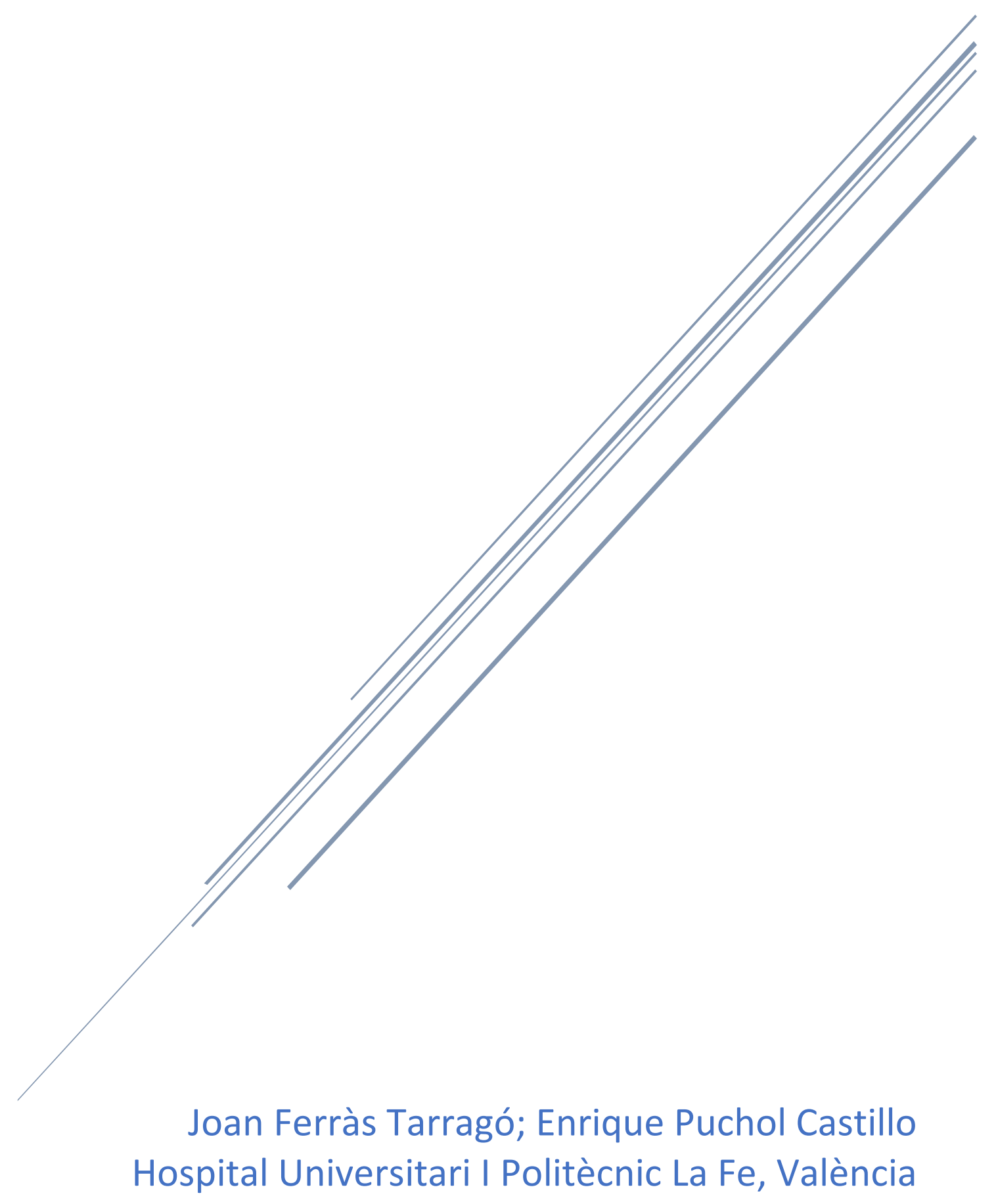




\section{Contenido}

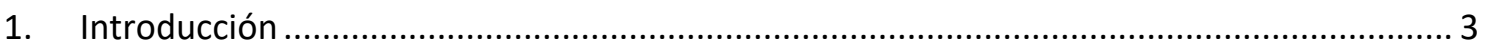

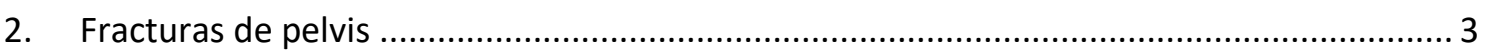

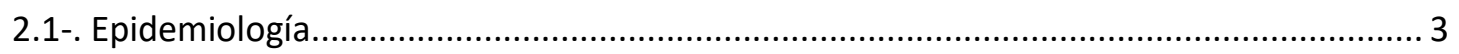

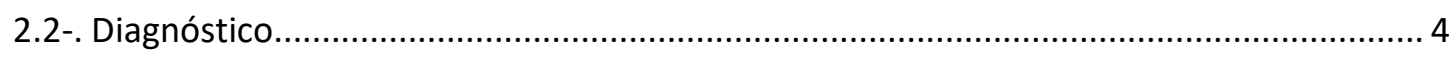

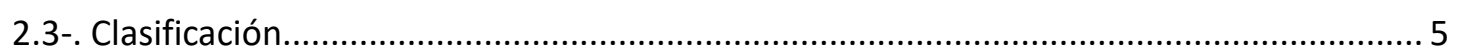

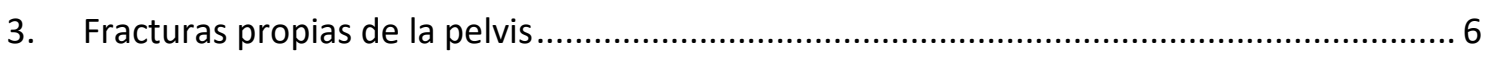

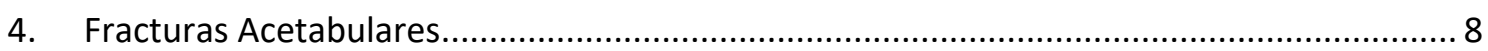

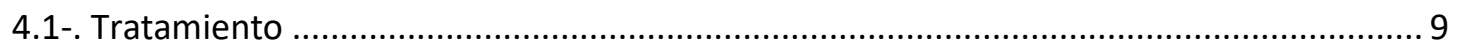

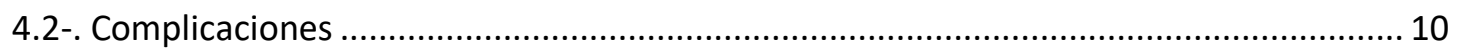

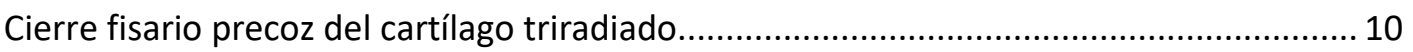

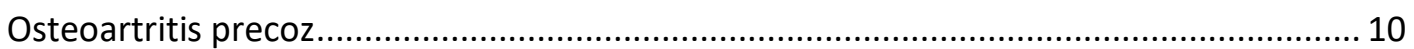

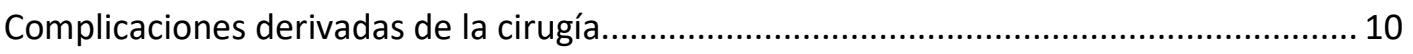

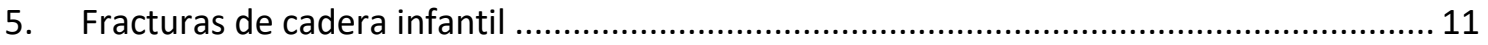

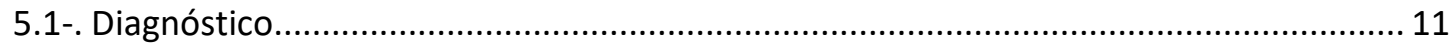

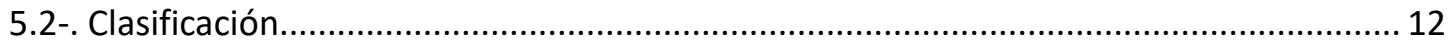

Anatomía vascular del extremo proximal del fémur......................................................... 12

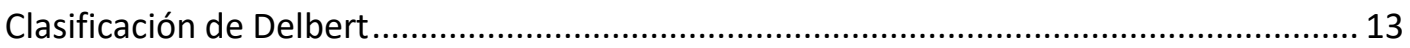

5.3-. Complicaciones: lo que tenemos que evitar .............................................................. 14

Necrosis Avascular de la cabeza del Fémur ..................................................................... 14

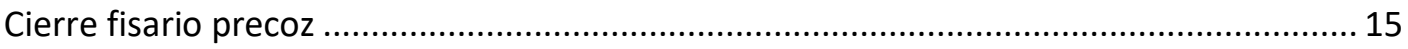

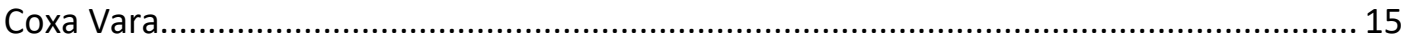

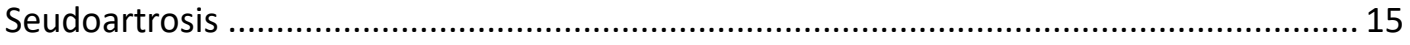

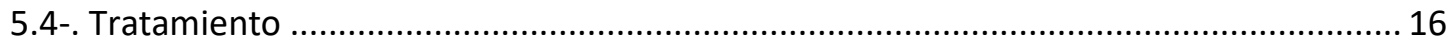

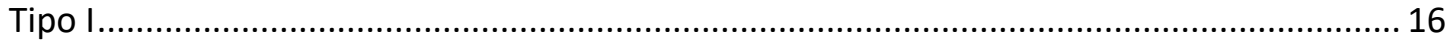

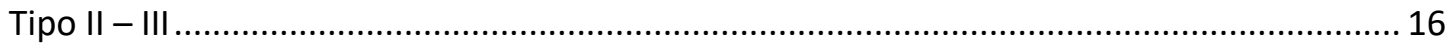

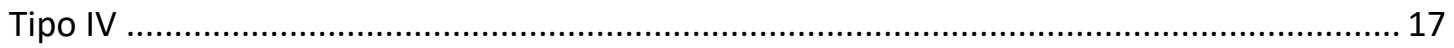

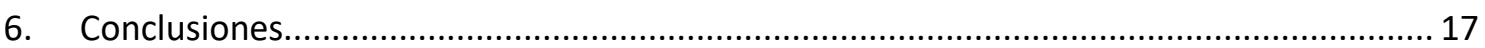




\title{
Fracturas Pélvicas y de Cadera Infantil
}

\author{
1. Introducción
}

En esta charla de la AAOs nos vamos a centrar en dos temas fundamentales:

- Fracturas de pelvis en la infancia

- Fracturas de cadera en la infancia

Se trata de una fractura rara (entre el $1 \%$ y el $3 \%$ de las fracturas en el caso de la pelvis y menos del $1 \%$ en el caso de fémur proximal), pero importante ya que conlleva consecuencias graves en caso de no tratarse correctamente.

Estas fracturas se caracterizan porque en la mayoría de ocasiones se producen en pacientes politraumatizados, con mucha otra patología vital asociada, y por lo tanto tenemos que diferenciar muy bien una primera atención de control de daños y un tratamiento definitivo. Siempre, lo más importante es el tratamiento de las lesiones asociadas de tejidos blandos, ya que son potencialmente fatales, dejando el tratamiento de la fractura en un segundo lugar.

\section{Fracturas de pelvis}

\section{1-. Epidemiología}

La fractura de pelvis pediátrica se caracteriza, como ya hemos contado, por producirse como consecuencia de traumatismos de alto impacto, siendo los accidentes de tráfico la principal causa. Por ello, es frecuente encontrar lesiones vasculares y nerviosas asociadas. El 50\% de esto niños asocian traumatismo craneoencefálico (TCE), y en función de la serie, asocian una mortalidad de un $25 \%$, aunque solo el $0,3 \%$ se relacionó con una exanguinación.

En función del desplazamiento de la fractura pélvica se asocian lesiones vasculares y nerviosas, siendo las arterias glúteas superior e inferior las más frecuentemente afectas a nivel de la escotadura ciática cuando se produce un desplazamiento en sentido cefálico. Cuando se produce una fractura en el anillo anterior, podemos encontrar lesiones de la arteria ilíaca o femoral, aunque es una situación rara.

Cuando sospechamos la afectación vascular, o bien mediante lavado peritoneal o bien mediante ecografía, se puede establecer el diagnóstico indirecto, y explorar mediante técnicas de imagen la arteria afecta de forma más selectiva. 
Cuando se produce un desplazamiento posterior en una fractura del anillo pélvico posterior, se puede encontrar afectación del nervio ciático, el plexo lumbosacro, el nervio femoral y el nervio obturador.

Cuando existe limitación para la micción, se debe de realizar una valoración por el especialista del aparato genitourinario, para descartar afectación del tracto, fundamentalmente en fracturas con inestabilidad vertical.

Las lesiones craneales son una de las principales causas de muerte, y su afectación puede ir desde la simple conmoción hasta la muerte cerebral.

Independientemente de las lesiones asociadas, la fractura de pelvis debe de tratarse como si el paciente fuera a recuperarse de sus lesiones, ya que las secuelas que produce un tratamiento incompleto son graves.

\section{2-. Diagnóstico}

El diagnóstico de la disrupción del anillo pélvico se basa en las radiografías en primera instancia. Éstas se deben obtener una vez estabilizado clínicamente le paciente, y se deben asociar radiografías de cráneo, tórax, abdomen, pelvis y huesos largos. Se debe practicar la proyección de inlet (a 25으 de angulación caudal) y outlet (35ㅇ de inclinación cefálica( para poder valorar el grado de desplazamiento anteroposterior y superoinferior respectivamente. La proyección oblícua nos puede dar información adicional. En caso de duda, o para la planificación quirúrgica, es útil la obtención de una tomografía computerizada (TC).

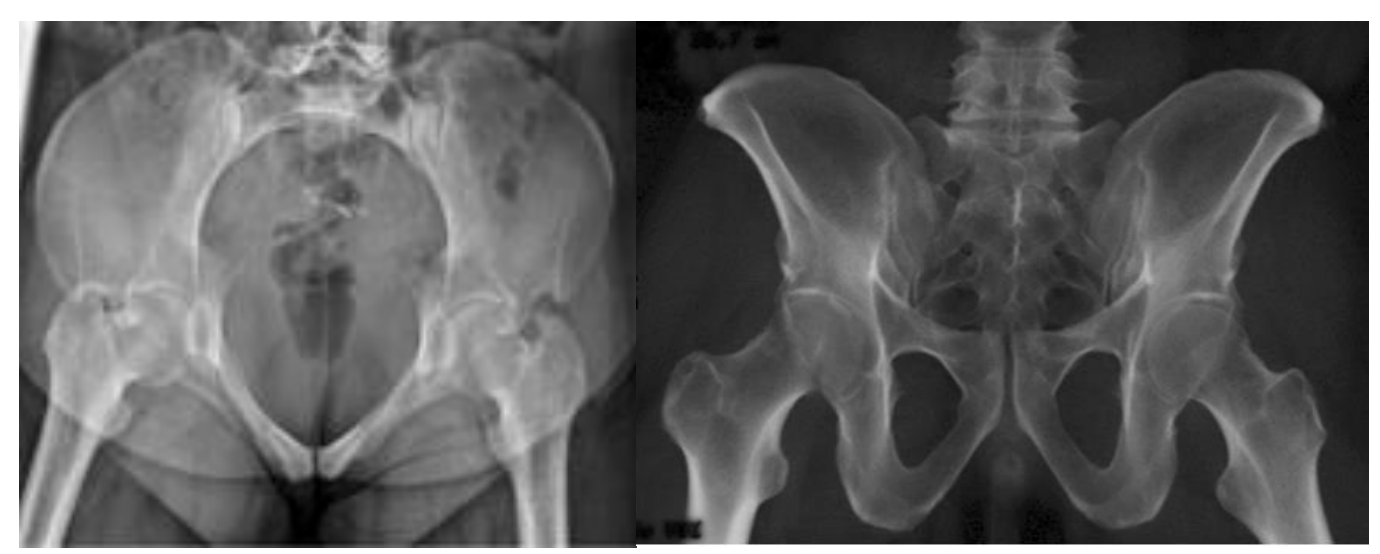

Ilustración 1. Proyección Inlet (izquierda) y outlet (derecha) de cadera 

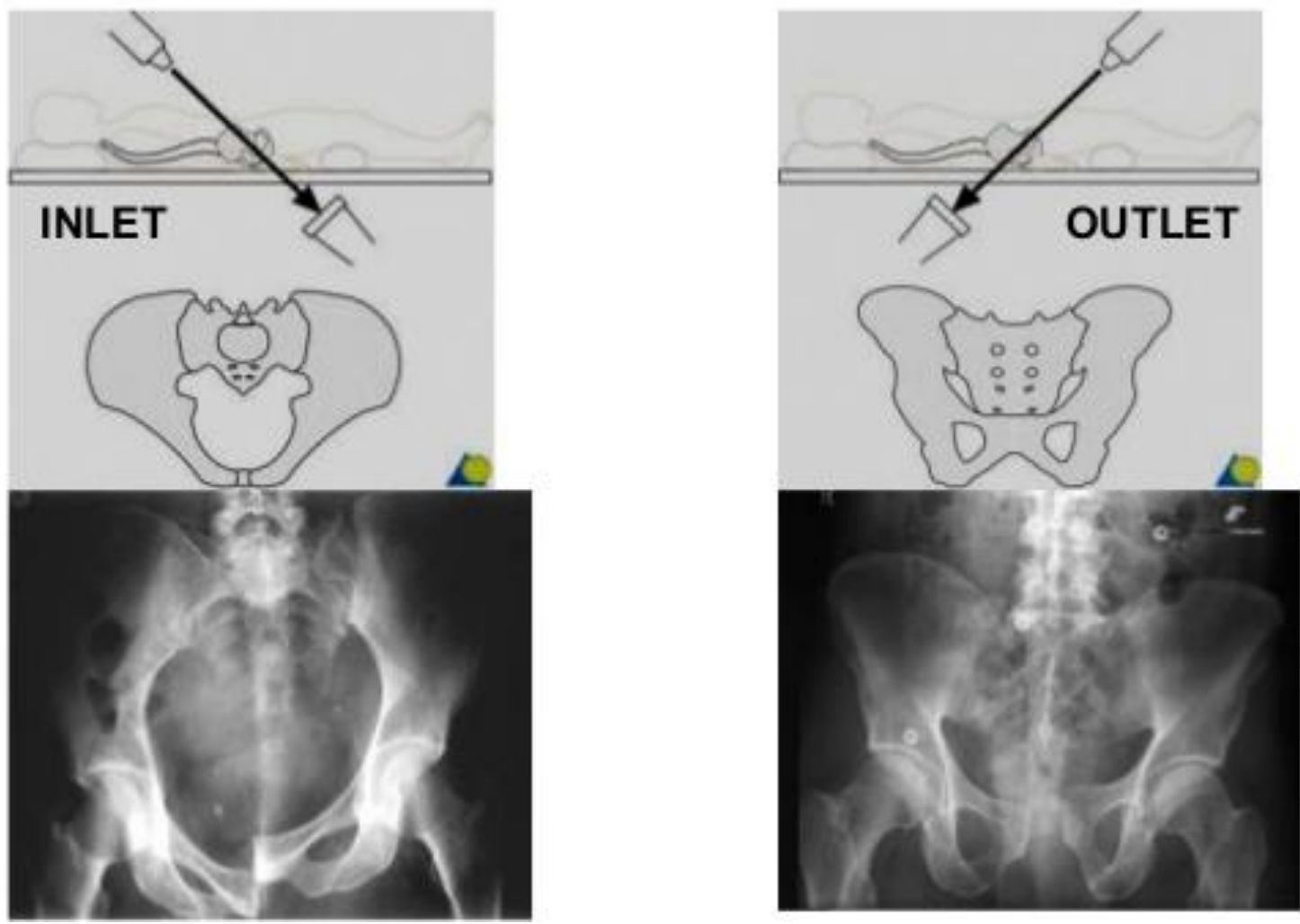

Ilustración 2. Modo de obtención de las radiografías diagnósticas en fracturas de pelvis. Tomado de Arbeitsgemeinschaft für Osteosynthesefragen (AO).

\section{3-. Clasificación}

Una vez realizada la estabilización clínica del paciente, y habiendo tomado las pruebas radiográficas complementarias, se puede clasificar la fractura pélvica de muchas formas diferentes, siguiendo diferentes criterios. Una de las más útiles por su practicidad es la clasificación de Quinby y Rang, que la clasifican en no complicada, con lesiones viscerales tributarias de exploración quirúrgica y fracturas asociadas a hemorragia masiva inmediata. Esta clasificación permite indicar al cirujano ortopédico pediátrico el momento de su entrada en escena en el cuadro de la atención al niño politraumatizado, y una vez dentro, la clasificación de la fractura simplemente en estable o inestable es suficiente para asentar indicación de tratamiento.

Las fracturas estables no requieren tratamiento quirúrgico urgente, y prácticamente nunca requieren tratamiento quirúrgico de forma programada. Al contrario, las fracturas inestables pueden requerir de la estabilización mediante sistemas auxiliares. Las principales fracturas inestables que asientan indicación de tratamiento adicional son:

- Dobles fracturas verticales de las ramas púbicas, también conocidas como fracturas flotantes.

- Dobles fracturas en el anillo pélvico al nivel anterior y posterior

- Múltiples lesiones por aplastamiento que asocien al menos dos fracturas conminutas en el anillo pélvico. 


\section{a) Fracturas a horcajadas}

En estas fracturas encontramos una lesión de dos ramas pélvicas anteriores ipsilaterales. La consecuencia es la generación de un fragmento flotante a nivel del anillo pélvico, que por norma general se encuentra desplazado en sentido ascendente debido a la tracción ejercida por los músculos abdominales.

Al tratarse de un fragmento que no está en contacto ni interpuesto con el eje de carga axial del cuerpo, no es necesario aplicar tracción al miembro afecto, y una mala reducción no asocia discrepancias de longitud de los miembros inferiores.

b) Fracturas a nivel anterior y posterior

Son las fracturas que más inestabilidad hemodinámica asocian, y por ello antes de ser tratadas, se debe estabilizar al paciente. Es fundamental medir la longitud del miembro afecto desde la espina ilíaca anterosuperior para el diagnóstico de este tipo de fractura, ya que nos proporciona una medida objetiva que nos debe llamar la atención.

Una vez estabilizado, generalmente se puede optar por un tratamiento conservador en la mayoría de los casos, reservando la fijación externa y la fijación interna únicamente para cuando el tratamiento conservador fracasa. El tratamiento conservador consiste en reposo en cama en decúbito lateral, y en el caso de que haya un ligero desplazamiento, se puede realizar manipulación cerrada en decúbito lateral y la implantación de un yeso pelvipédico. Si hay un desplazamiento cefálico, se puede usar la tracción tranesquelética.

Los niños raramente sangran, la seudoartrosis es rara, tienen un periostio grueso que mantiene la reducción, no tienen que inmovilizarse mucho tiempo y remodelan muy bien, por lo que la indicación de la fijación ya sea interna o externa, es rara en este tipo de fracturas.

Cuanto más grande sea el niño, más mala tolerancia al yeso presenta y menos ventajas del hueso infantil asocia, por lo que más indicación de fijación similar al adulto se tiene que tomar en cuenta.

En ambos tipos de fractura, cuando hay un desplazamiento mayor de $3 \mathrm{~cm}$ en la articulación sacroilíaca con tratamiento conservador (reducción cerrada + yes / tracción ), se asienta indicación quirúrgica mediante reducción abierta y fijación interna, siendo a partir de 8-10 años de edad cuando se establece el límite entre niño/adolescente.

\section{Fracturas propias de la pelvis}

Las fracturas propias de la pelvis son fracturas poco frecuentes y que, a excepción de las fracturas pélvicas del paciente politraumatizado, se pueden resumir en su práctica totalidad al tratamiento conservador con reposo y deambulación con apoyo ponderal progresivo. Eso es debido a que son más elásticas que las del adulto, y se puede romper por una parte del anillo sin que se tenga que romper por dos. En niños muy pequeños que toleren el yeso pelvipédico se puede utilizar para acelerar la recuperación y reducir el dolor hasta que se produce. 
Las fracturas aisladas de huesos de la pelvis son:

- Fractura de cóccix

- Fractura de sacro

- Fractura de ala ilíaca

- Fractura de ramas

- Fracturas proximales al pubis

- Fracturas proximales a las articulaciones sacroilíacas

Un tipo más particular de fracturas pélvicas durante la infancia y adolescencia son las fracturas avulsión. Éstas son más frecuente en las primeras etapas de la vida, fundamentalmente durante la adolescencia en deportes que impliquen dar patadas, por el mecanismo de contracción violento que asocia este movimiento.

El motivo por el que le dedicamos más tiempo que al resto de las fracturas propias de la pelvis es porque se pueden confundir con uno de los núcleos de osificación secundarios de la pelvis en un niño que presenta simplemente una pequeña entesopatía de estos músculos. El diagnóstico diferencial entre una entesopatía en un niño que tiene núcleo de osificación secundario y una fractura avulsión es difícil, y se realiza en función de la intensidad de los síntomas y de la evolución. Para salir de dudas, podemos realizar una radiografía de pelvis completa y observar si la misma anomalía se encuentra en la hemipelvis asintomática.

La principal ventaja que tienen estas pequeñas fracturas es que se tratan de forma conservadora impidiendo la acción de estos músculos, al igual que la entesopatía, y lo único que tarda será el tiempo durante el que presentará sintomatología.

La única indicación quirúrgica de estas fracturas es en los extrañísimos casos de pseudoartrosis, en los cuales se opta por la reducción interna.

Debido a la importancia de los núcleos de osificación secundario, realizamos un ejercicio práctico para su análisis. Dejo la resolución en el siguiente esquema.

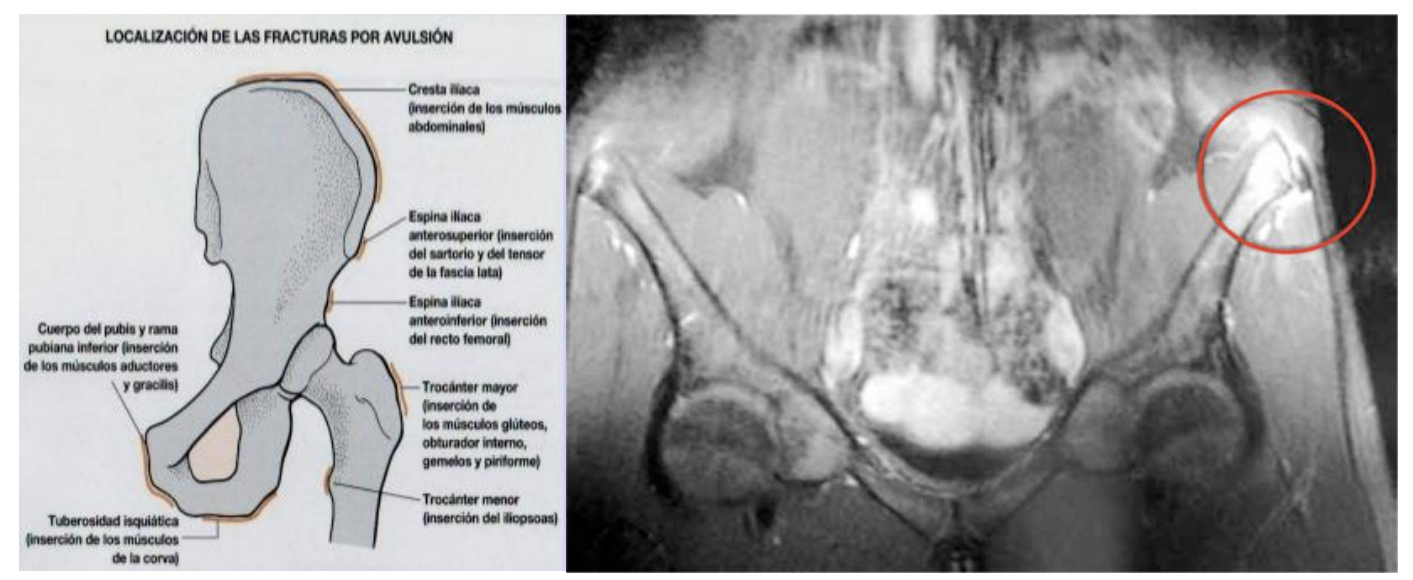




\section{Fracturas Acetabulares}

Siguen siendo fracturas asociadas a traumatismos graves, que a menudo se asocian a fracturas de fémur o de otras partes de la pelvis, por lo que seguimos teniendo a un paciente en el que la estabilización clínica vital será nuestra prioridad.

Estas fracturas suelen producirse a consecuencia del impacto de la cabeza femoral en el acetábulo, de forma que la fractura queda determinada por:

- La forma de la fractura la determina la posición de la cabeza femoral en el impacto

- El grado de desplazamiento lo determina la intensidad de la fuerza deformante.

El diagnóstico se establece con las mismas proyecciones que para el diagnóstico de fracturas de pelvis, pero en esta ocasión las proyecciones oblicuas de Judet (45 de oblicuidad lateral) aumentarán su importancia. Aún con estas proyecciones, es fácil que pasen desapercibidas, por lo que los signos indirectos de fractura son fundamentales:

- Aumento del espacio articular secundario al hemartros intraarticular

- Disrupción de la línea de Shenton

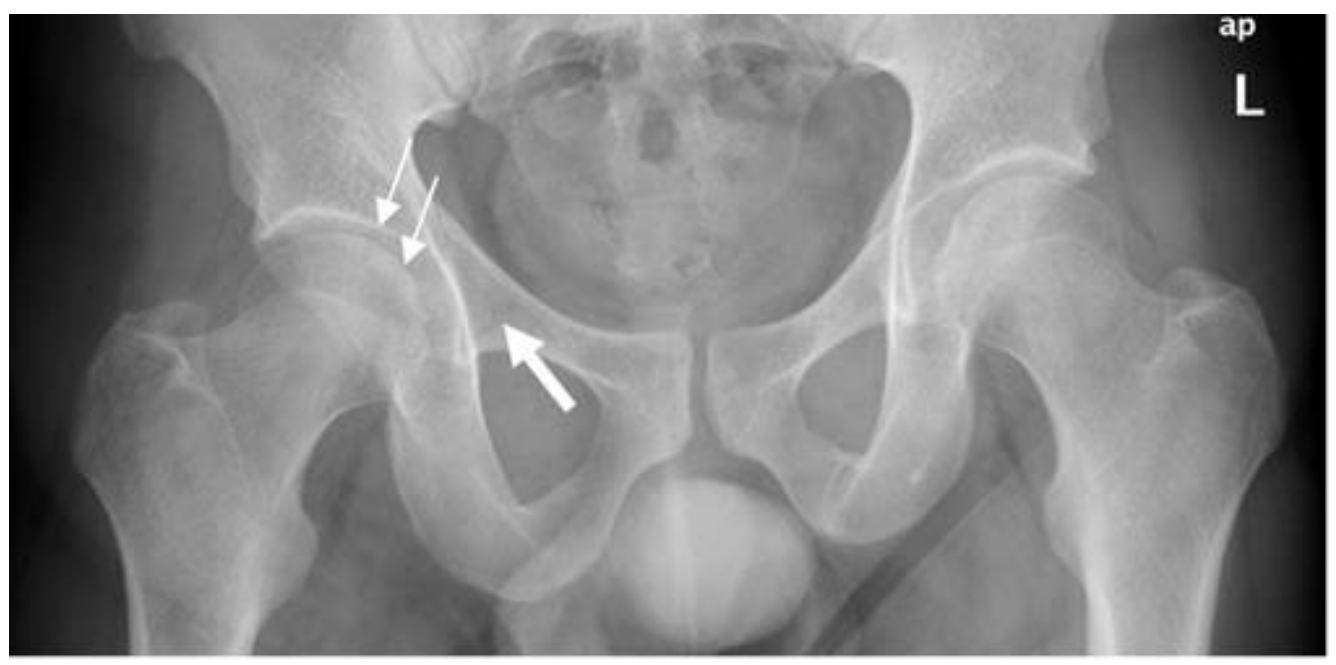

Ilustración 3. Imagen en la que observamos disrupción de la Línea de Shenton en contexto de fractura acetabular, visible en la radiografía pero con gran dificultad.

Los signos secundarios nos sirven para ampliar los estudios con imágenes más agresivas como la tomografía computerizada, que es el gold standard tanto para la toma de la decisión del tratamiento definitivo como para la planificación quirúrgica.

Mediante las pruebas de imagen, se puede clasificar, siendo la de la AO la principal clasificación a seguir para la decisión de tratamiento definitivo. Debido a la existencia del cartílago triradiado a nivel acetabular, la clasificación de Salter y Harris es también posible, considerándose la tipo $V$ aquella fractura que se produce por un mecanismo de compresión. 


\section{1-. Tratamiento}

La articulación de la cadera es una de las más importantes del organismo, ya que de no conseguir una reducción perfecta desarrolla osteoartritis precoz. Es por ello que la fractura acetabular en la infancia es una de las pocas que se rige por los mismos principios que el tratamiento de las fracturas en el adulto.

Podemos conseguir la reducción anatómica de la forma que queramos (entre comillas), pero independientemente de la edad tenemos que conseguirlo. Consideramos una reducción anatómica como aquella que mediante un sistema conservador consigue un desplazamiento articular menor de $\mathbf{2} \mathbf{~ m m}$. Ello significa que, o bien sin mecanismo de tracción, o bien con un mecanismo de tracción transesquelética con una AK en el fémur, podemos no hacer reducción abierta si se consigue menos de $2 \mathrm{~mm}$ de escalón articular. En niños mayores, el mantenimiento de una tracción transesquelética durante 6 semanas es difícil, motivo por el que en ellos se indica la reducción abierta y fijación interna cuando hay un escalón inicial de 2 $\mathrm{mm}$. En niños pequeños, como disponemos de la opción de la tracción, podemos intentarlo antes de realizar reducción abierta (menores de 6-8 años). Si optamos por un tratamiento simplemente conservador, se deberá mantener en descarga durante el mismo tiempo, y a partir de ahí podremos empezar a realizar carga progresiva.

La fijación interna de una fractura acetabular es sin duda un reto quirúrgico, que solo se debe realizar por expertos en la materia. El principal abordaje utilizado es el de Kocher-Langenbeck (posterior), a no ser que se trate de una fractura aislada de la pared anterior, en cuyo caso, estaría indicado un abordaje de Smith-Petersen (anterior) o bien un abordaje Ilioinguinal. Los abordajes lateral ampliado (iliofemoral ampliado y trirradiado) deben evitarse por el riesgo de desvascularización del ilíaco y la osificación heterotópica.

Para la reducción una vez hemos realizado el abordaje, es posible tener que completar la fractura para conseguir una reducción anatómica. La estabilidad la daremos mediante el sistema de fijación, y si no rompemos la osteotomía adecuada (pubis, ilíaco o isquion en función de la fractura), no se conseguirá la reducción adecuada.

Es importante, sobre todo en niños pequeños, realizar la intervención con la mayor brevedad posible siempre que presente una situación clínica favorable, ya que pasadas las $24 \mathrm{~h}$ la reducción es más compleja debido al alto potencial de osificación que presentan estos pacientes.

Una vez intervenido, se debe realizar la extracción del material de osteosíntesis pasados 6 a 18 meses tras la cirugía si el paciente sigue con el cartílago de crecimiento abierto ya que de lo contrario, el material de osteosíntesis quedará incluido en el espesor del hueso. Teniendo en cuenta el riesgo de osteoartritis precoz y la necesidad de la artroplastia de sustitución precoz, es importante que el material de osteosíntesis no suponga un impedimento para esta cirugía, de ahí la importancia de ser previsores y retirarlo antes de que para ello requiramos osteotomías y una cirugía mucho más agresiva. 


\section{2-. Complicaciones}

Las dos principales complicaciones derivadas de una fractura acetabular en la edad infantil son:

- El cierre fisario precoz del cartílago triradiado

- La osteoartritis precoz

Cierre fisario precoz del cartílago triradiado

Se produce por la lesión de la fisis durante la fractura. El acetábulo crece por aposición intersticial en la parte triradiada del complejo cartilaginoso. Si se produce una lesión del cartílago, se produce un crecimiento asimétrico del acetábulo lo que conlleva a una alteración en la profundidad y la forma normal del mismo, y por lo tanto, a una displasia acetabular que puede llevar a dismetrías y a subluxaciones de la cadera afecta. Cuando esto ocurre, se debe realizar una resección del puente óseo con la mayor brevedad posible, para evitar el crecimiento displásico del acetábulo y minimizar así las consecuencias del cierre fisario, sobretodo en menores de $\mathbf{1 0}$ años.

\section{Osteoartritis precoz}

La osteoartritis precoz es una consecuencia del fallo en la reconstrucción acetabular tras la fractura y de un crecimiento anormal del acetábulo. Por desgracia, son pocas las opciones de las que disponemos cuando aparece, siendo la principal finalidad el retraso en la implantación de la artroplastia de sustitución en la medida de lo posible.

\section{Complicaciones derivadas de la cirugía}

Son dependientes de las pautas quirúrgicas que realicemos, y aunque lo hagamos fenomenal, siempre hay riesgo de que aparezcan. Las principales son la parálisis del nervio ciático (también relacionada con el momento de producción de la fractura), la miositis osificante heteropópica y la distocia del parto. 


\section{Fracturas de cadera infantil}

De nuevo, nos encontramos ante una fractura de baja prevalencia, pero que aprovecharemos para comprender el funcionamiento y la biomecánica de la articulación de la cadera pediátrica. La ventaja de estudiar estas fracturas es que aprendemos qué estructuras se pueden lesionar de una forma traumática y sus consecuencias. Esto no es importante solo para la comprensión de las fracturas de cadera, sino que nos sirve mucho para saber qué no tenemos que lesionar cuando realizamos intervenciones sobre el fémur proximal.

Por lo tanto, aprenderemos de las consecuencias que tiene la lesión traumática accidental sobre el fémur proximal para conocer su funcionamiento y así aprender no solo el manejo del traumatismo agudo, sino también saber las consecuencias que pueden tener nuestros actos sobre una articulación tal importante como esta.

Se trata de fracturas raras, con una incidencia inferior al 1\% de todas las fracturas de los niños. Una vez más, se trata de fracturas asociadas a mecanismos de alta energía, por lo que de nuevo debemos considerar la estabilidad clínica del paciente antes que la resolución de la fractura.

\section{1-. Diagnóstico}

Generalmente se trata de fracturas de alto impacto, por lo que acudirán con una sospecha clínica alta: dolor en cadera tras traumatismo de alta energía. Se realizará de forma rutinaria radiografías anteroposteriores de pelvis, para descartar fracturas pélvicas o de cadera, y generalmente ahí se verán. El paciente además asociará dolor y limitación fundamentalmente para la rotación interna.

Cuando hay duda diagnóstica en la radiografía, se puede solicitar una proyección axial de la cadera afecta, sabiendo que se debe restringir si el diagnóstico es claro por el riesgo de provocar el desplazamiento de la fractura obteniendo la radiografía. La zona más sensible para la valoración de una posible fractura es el triángulo de Ward, espacio de debilidad que queda entre las líneas de tracción y de compresión a las cargas del fémur proximal.

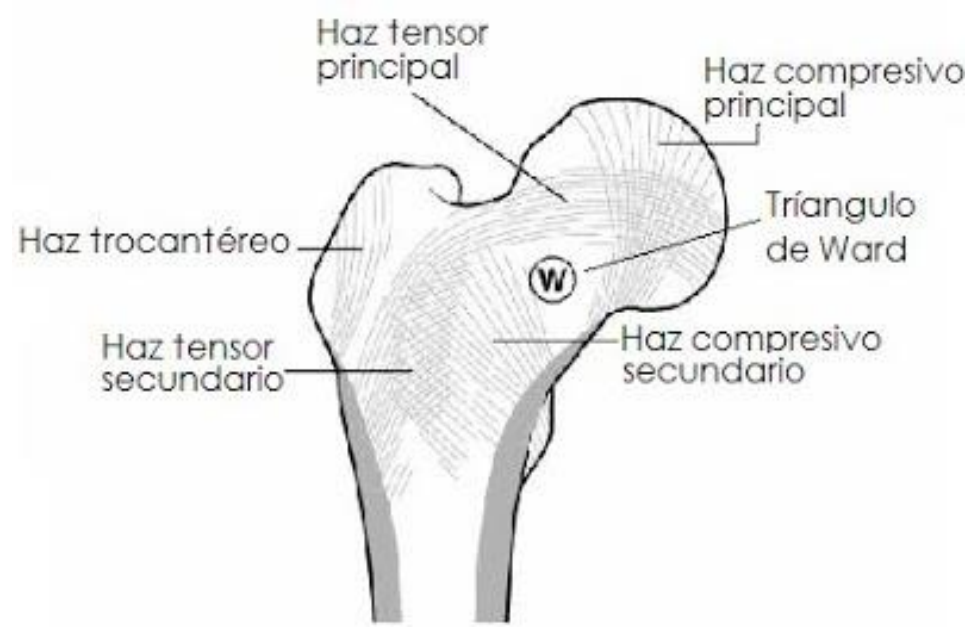

Ilustración 4. Triángulo de Ward y haces de compresión y de tensión del fémur proximal. 
La resonancia magnética nuclear (RMN) y la gammagrafía (muy infrecuente desde la aparición de la RMN) son útiles para la valoración de fracturas que pasan desapercibidas en la radiografía y para el estudio de fracturas de estrés.

Cuando el mecanismo lesivo no ha sido de alta energía y el paciente presenta dolor de cadera de origen dudoso, la artrocentesis de la cadera actúa como prueba diagnóstica. El diagnóstico diferencial de una cadera dolorosa en el niño comprende:

- Sinovitis

- Artritis séptica

- Hemartros

- Fractura

El hemartros y la fractura presentarán un aspirado sanguinolento, la sinovitis un aspirado seroso y la artritis séptica un aspirado purulento, claro está, en condiciones ideales. La analítica sanguínea incluyendo Proteína C Reactiva (PCR) y Velocidad de Sedimentación Globular (VSG), así como bioquímica y hemograma con recuento leucocitario son de gran utilidad en el diagnóstico diferencial.

Con esto, podemos pasar a la clasificación pronóstica de la fractura de Ratliff

\section{2-. Clasificación}

No podemos comprender las clasificaciones de las fracturas de fémur proximal sin antes estudiar su anatomía vascular, ya que éstas suelen establecer la gradación en función del riesgo de su desarrollo.

\section{Anatomía vascular del extremo proximal del fémur}

Los vasos que nutren la cabeza femoral surgen de la arteria femoral circunfleja medial y de la arteria circunfleja femoral lateral, siendo la medial la principal encargada de ello. De la circunfleja medial surge la arteria ascendente lateral cerfical , y su rama posterior-superior irriga la porción superior de la epífisis femoral. La rama posterior inferior, también rama de la circunfleja medial se encarga de la parte más medial de la epífisis femoral. Aparte, de la arteria circunfleja medial surgen vasos metafisarios, que se encargan de nutrir la epífisis.

Conforme avanza el tiempo, el conjunto de vasos epifisarios van confluyendo en ramas más gruesas y más individuales, siendo capaces de identificar más claramente por zonas la irrigación de la cabeza femoral. 
Con esto, podremos comprender el motivo por el cual las complicaciones de los diferentes grados de la clasificación de la fractura de fémur proximal van cambiando conforme bajamos el nivel de la fractura.

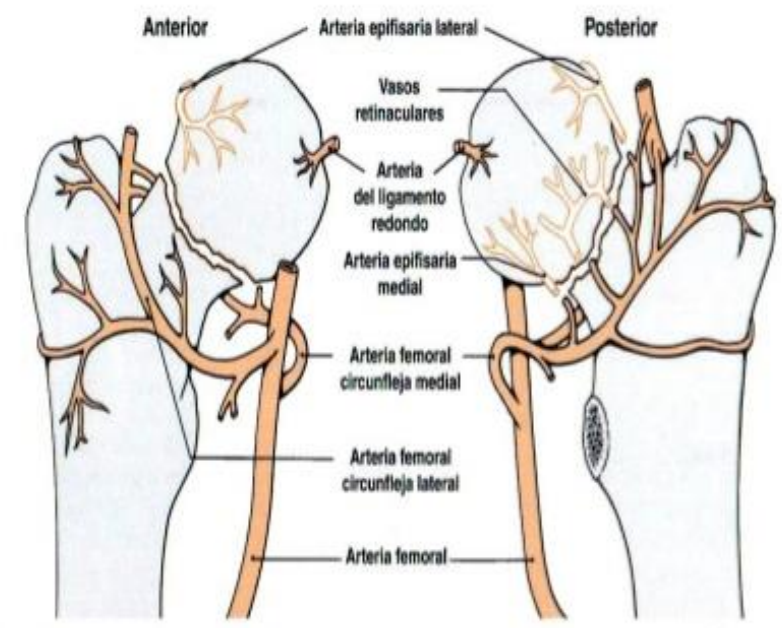

Ilustración 5. Anatomía vascular del extremo proximal del fémur.

\section{Clasificación de Delbert}

La clasificación de Delbert de las fracturas de fémur proximal es la más utilizada por su simplicidad y su correlación con la gravedad. La complicación más grave de la fractura de fémur proximal es la osteonecrosis, que estudiaremos más adelante, y la clasificación de Delbert escala descendentemente el griesgo de necrosis avascular (NAV) conforme progresan los grados de su clasificación. Establece los siguientes grados:

- Tipo I: Epífisis femoral proximal traumática. El riesgo de necrosis avascular es muy alto, cerca del $100 \%$, así como del cierre fisario precoz. Se diferencia de la epifisiolisis femoral proximal por la característica traumática de este tipo.

- Tipo II: Fractura transcervical. Cuanto menos desplazamiento presente, menos riesgo de NAV asocia. Una particularidad de este tipo de fractura es que se ha descrito que al ser intraarticular, pero estar por debajo de los vasos epifisarios, el aumento de la presión intraarticular asociada al sangrado en el foco de fractura puede ser el responsable de la aparición de NAV en este tipo de fracturas, más que la lesión de los vasos nutricios. Por ello, es un tipo de fractura que se beneficia más que el resto de la artrocentesis de evacuación, con la finalidad de reducir la presión intraarticular y reducir el colapso asociado de los vasos epifisarios.

- Tipo III: Fractura cervicotrocantéreas. Están a nivel o por encima del surco intertrocantéreo. En este tipo el riesgo de NAV se reduce al $20 \%-30 \%$, y es mayor cuanto mayor desplazamiento inicial asocian, similar a lo que ocurre con el tipo II.

- Tipo IV: Intertrocantéreas. Son las más benignas de su clase, ya que no asocian prácticamente riesgo de NAV, seudoartrosis ni coxa vara. 
Una vez entendemos la clasificación, y sobretodo, entendemos por qué se clasifica de esta forma, podemos dar paso al estudio del tratamiento, que será de una forma u otra en función de lo que podamos ganar vs lo que podamos perder interviniendo o no al paciente.

\section{3-. Complicaciones: lo que tenemos que evitar}

Me parece adecuado ver las complicaciones antes que el tratamiento, ya que en este tipo de fracturas el tratamiento está dirigido fundamentalmente a esto: a reducir las complicaciones. Concretamente, a reducir la NAV.

\section{Necrosis Avascular de la cabeza del Fémur}

El 30\% de las fracturas de fémur proximal asocia NAV, y el riesgo es mayor en función del grado de desplazamiento inicial. Ya hemos estudiado la anatomía vascular de la cabeza femoral, por lo que nos será sencillo comprender la principal clasificación de esta complicación: la clasificación de Ratliff.

La clasificación de Ratliff es la más utilizada en cuanto a la osteonecrosis del extremo proximal de fémur. El motivo es que supone una gradación de la severidad y riesgo de complicaciones de la fractura, que como veremos, está justificada por la anatomía vascular de la extremidad proximal del fémur.

En la clasificación de Ratliff, encontramos:

- Tipo I. Se produce por la lesión de los vasos circunflejos femorales, lo que produce una lesión de prácticamente toda la cabeza femoral, tanto de la metáfisis como de la epífisis.

- Tipo II. Se produce una lesión de los vasos epifisarios, ya sean las ramas posteriores y superiores o bien sean los vasos posteriores e inferiores, lo que produce afectación de una parte de la epífisis femoral

- Tipo III: Se afectan los vasos metafisarios, por lo que se produce una necrosis metafisaria respetando la porción epifisaria.

Aunque esta clasificación se basa en la necrosis en función de la disrupción de los vasos nutricios, hemos visto que hay otro factor que influye en el aporte sanguíneo tras una fractura de fémur proximal: la presión intraarticular. Cuando la presión es muy alta, se pueden colapsar los vasos epifisarios y metafisarios intracapsulares. Es por ello que la artrocentesis y evacuación del hemartros ha demostrado reducir el riesgo de NAV.

El diagnóstico se basa en la sospecha clínica, con persistencia del dolor y la limitación más tiempo de lo habitual tras la reducción y fijación de la fractura. Se confirma con una radiografía a las 6 semanas (o más tarde), donde se puede objetivar una reducción de la densidad radiográfica, con ensanchamiento del espacio articular, aunque esto puede aparecer durante 
los dos primeros años tras el traumatismo, por lo que está indicado el seguimiento radiográfico durante este período de tiempo.

Cuando aparece, el $60 \%$ de los pacientes quedan insatisfechos, y no hay un tratamiento claramente eficaz, siendo peores los resultados cuanto más mayor sea el niño, tanto por la reducción en la capacidad de remodelación como por la agrupación de los vasos sanguíneos.

La complicación a largo plazo es la osteoartritis precoz, y en una fase temprana se puede intentar reducir el riesgo mediante la descarga del miembro. La descompresión nuclear, los injertos vascularizados de peroné y el procedimiento de la trampilla, son nuevos tratamientos que intentan mejorar los resultados, pero son necesarios más estudios y de mayor calidad para demostrarlo.

\section{Cierre fisario precoz}

Se produce también en el $30 \%$ de los pacientes aproximadamente, y a menudo se asocia a los procedimientos quirúrgicos de fijación para prevenir el desarrollo de la NAV.

La realidad es que sus consecuencias son menos graves que la NAV, por eso a veces preferimos sobrepasar la fisis femoral proximal y asegurar una buena fijación, asumiendo el riesgo de desarrollar un cierre fisario. Dado que solo es responsable del $13 \%$ de la longitud del miembro, solo se deberá intentar su corrección cuando se prevé una dismetría mayor a 2,5 cm tras finalizar el período de crecimiento.

La coxa vara es una de sus consecuencias, ya que de persistir creciendo por la fisis trocantérea, y no crecer por la fisis femoral proximal, se produce una varización progresiva, que en caso de ser muy llamativa, puede requerir realizar una epifisiodesis trocantérea.

\section{Coxa Vara}

Se produce en el $20-30 \%$ de los pacientes, y es una consecuencia de muchas de las complicaciones que se asocian a esta fractura: NAV, cierre fisario precoz, consolidación defectuosa o una mezcla de todas ellas. Las consecuencias directas son un acortamiento del miembro y una reducción en la fuerza de los abductores, con la consecuente marcha en bamboleo abductor. Los criterios de una osteotomía subtrocantérea valguizante son:

- Más de 8 años de edad, ya que no se espera que remodele

- Ángulo cervicodiafisario mayor de 110은

- Persistencia de al menos 2 años de dicha deformidad.

\section{Seudoartrosis}

Es extremadamente rara, y solo la comentamos por el hecho de que se diagnostica con un retraso de consolidación de 3 meses, y para su tratamiento simplemente se debe realizar una fijación, sin aporte de injerto. 


\section{4-. Tratamiento}

El tratamiento maneja la fina balanza entre el riesgo de producir un cierre fisario precoz y el riesgo de desarrollar NAV si se deja sin fijación estable. Por ello, en el grado I deberemos ser más estrictos en la fijación, aunque produzcamos con ello un cierre fisario precoz. A modo general tenemos que recordar que los sistemas de fijación son:

- Para menores de 3 años: AK

- Entre 3 y 8 años: Canulados de 4,5 mm

- Mayores de 8 años: Canulados de 6,9 mm

En todos los casos, antes de introducir los tornillos se debe perforar y puncionar, ya que tienen una calidad ósea mucho mayor que los adultos y por lo tanto la introducción de tornillos sin perforación previa es muy lesiva para el hueso.

Tipo I

En estas fracturas, siempre que haya que fijarlas, la fijación deberá pasar la fisis, y a los 8-12 meses se deberá retirar la fijación, sea la que sea, para reducir el riesgo de cierre fisario precoz.

En los mayores, debido al alto riesgo de NAV que presentan (porque recordemos que los vasos han empezado a juntarse), siempre habrá que fijarlas. La forma de fijación podrá ser con AK o con tornillos, y generalmente se establece la edad de 8 años como límite.

En niños pequeños, menores de $\mathbf{2}$ años y fracturas sin desplazar se puede optar por un yeso pelvipédico. La realidad es que se opta por ello porque es una forma tolerada de fijar la fractura, el problema es que tienen que ser niños muy pequeños que puedan soportar el yeso unas 6 semanas, y eso más allá de 2 años es muy difícil. En el caso de estar desplazadas implica que son inestables y por lo tanto habrá que fijarla, y como son pequeños, se podrá hacer con AK. Además, el desplazamiento inicial es el principal predictor de NAV, motivo de más para fijar la fractura con AK aun siendo pequeño si hay desplazamiento inicial.

Tipo $\|-\|$

En estas fracturas la fijación no tiene necesidad de atravesar la fisis. En caso de ser inestable sin atravesarla, se debe atravesar, ya que el riesgo de NAV siempre es menos asumible que el de cierre fisario. Las dos principales complicaciones en estas fracturas si no se fijan son la seudoartrosis y la coxa vara, por eso, haya o no haya desplazamiento, se debe fijar según el estándar explicado al principio, siendo más agresivo o menos a la hora de atravesar la fisis en función de su estabilidad. En las más distales, se puede usar un tornillo de compresión dinámica (DHS) pediátrico.

La reducción se consigue con tracción, abducción y rotación interna, y la configuración de la fijación debe de ser en triángulo invertido, con la máxima distancia de separación entre ellos que sea posible conseguir. 
Si los niños son pequeños, se implanta un yeso pelvipédico, y si son mayores, confiamos en que sean responsables y que no carguen.

Como siempre que amenazamos la fisis, se debe retirar el implante pasados unos 6-12 meses tras la fijación.

Tipo IV

Independientemente de la edad, si no está desplazada o se consigue una reducción cerrada satisfactoria (fundamentalmente mediante tracción), se puede optar por tratamiento conservador con descarga en el caso de niños mayores y con yeso pelvipédico en el caso de que el niño sea pequeño y lo tolere. En caso de pacientes politraumatizados, o en aquellos que no se consiga la reducción, se optará por la fijación, que en el caso de mayores de $\mathbf{8}$ años se realizará mediante un tornillo de adulto, y en aquellos menores de 8 años se optará por un clavo pediátrico.

\section{Conclusiones}

Hemos realizado una revisión de las fracturas pélvicas y de cadera en la edad infantil, que nos servirá no solo para adoptar una actitud correcta en estas fracturas, sino que nos servirá también para comprender las principales lesiones y complicaciones asociadas a la intervención sobre estas estructuras. La cirugía sobre la cadera y pelvis infantil es una cirugía compleja, con una gran morbilidad y con muchas complicaciones derivadas de la necrosis y de la falta de consolidación, además de por la afectación de las fisis de crecimiento. En esta charla hemos usado los traumatismos naturales para comprender las consecuencias derivadas de nuestros traumatismos sobre las mismas estructuras, y así mejorar nuestra pericia traumatológica y también durante la cirugía ortopédica. 